\title{
Accretion onto some well-known regular black holes
}

\author{
Abdul Jawad $^{1, \mathrm{a}}$, M. Umair Shahzad ${ }^{1, \mathrm{~b}}$ \\ ${ }^{1}$ Department of Mathematics, COMSATS Institute of Information Technology, Lahore 54000, Pakistan
}

Received: 19 January 2016 / Accepted: 17 February 2016 / Published online: 7 March 2016

(C) The Author(s) 2016. This article is published with open access at Springerlink.com

\begin{abstract}
In this work, we discuss the accretion onto static spherically symmetric regular black holes for specific choices of the equation of state parameter. The underlying regular black holes are charged regular black holes using the Fermi-Dirac distribution, logistic distribution, nonlinear electrodynamics, respectively, and Kehagias-Sftesos asymptotically flat regular black holes. We obtain the critical radius, critical speed, and squared sound speed during the accretion process near the regular black holes. We also study the behavior of radial velocity, energy density, and the rate of change of the mass for each of the regular black holes.
\end{abstract}

\section{Introduction}

At present, the type 1a supernova [1], cosmic microwave background (CMB) radiation [2], and the large scale structure $[3,4]$ have shown that our universe is currently in an accelerating expansion period. Dark energy is responsible for this acceleration and it has the strange property that it violates the null energy condition (NEC) and the weak energy condition (WEC) [5,6] and produces strong repulsive gravitational effects. Recent observations suggests that approximately $74 \%$ of our universe is occupied by dark energy and the rest 22 and $4 \%$ is of dark matter and ordinary matter, respectively. Nowadays dark energy is the most challenging problem in astrophysics. Many theories have been proposed to handle this important problem in last two decades. Dark energy is modeled using the relationship between energy density and pressure by a perfect fluid with the equation of state (EoS) $\rho=\omega p$. The candidates of dark energy are a phantomlike fluid $(\omega<-1)$, quintessence $(-1<\omega<-1 / 3)$, and the cosmological constant $(\omega=-1)$ [7]. Other models are also proposed as an explanation of dark energy, like

\footnotetext{
a e-mails: jawadab181@yahoo.com; abduljawad@ciitlahore.edu.pk

be-mail: m.u.shahzad@ucp.edu.pk
}

k-essence, DBI-essence, Hessence, dilation, tachyons, Chaplygin gas, etc. [8-16].

On the other hand, the existence of essential singularities [which leads to various black holes (BHs)] is one of the major problems in general relativity $(\mathrm{GR})$ and it seems to be a common property in most of the solutions of Einstein's field equations. To avoid these singularities, regular BHs (RBHs) have been developed. These BHs are solutions of Einstein's equation with no essential singularity; hence their metric is regular everywhere. The strong energy condition (SEC) is violated by these RBHs somewhere in space-time [17,18], while some of these satisfy the WEC. However, it is necessary for those RBHs to satisfy the WEC having a de Sitter center. The study of an RBHs solutions is very important for understanding the gravitational collapse. Since the Penrose cosmic censorship conjecture claims that singularities predicted by GR $[19,20]$ occur, they must be explained by event horizons. Bardeen [21] has done pioneering work in this way by presenting the RBH known as the "Bardeen black hole", satisfying the WEC.

The discussion as regards the properties of the BHs have led to many interesting phenomena. Accretion onto the $\mathrm{BHs}$ is one of them. When massive condensed objects (e.g. black holes, neutron stars, stars etc.) try to capture a particle of the fluid from its surroundings, then the mass of condensed object has been effected. This process is known as accretion of fluid by condensed object. Due to accretion the planets and star form inhomogeneous regions of dust and gas. Supermassive BHs exist at the center of giant galaxies, which suggests that they could have formed through an accretion process. It is not necessary that the mass of the $\mathrm{BH}$ increases due to the accretion process, sometimes in-falling matter is thrown away like cosmic rays [22]. For a first time, the problem of accretion on a compact object was investigated by Bondi using the Newtonian theory of gravity [23]. After that many researchers such as Michel [24], Babichev et al. [25,26], Jamil [27] and Debnath [31] have discussed the accretion on Schwarzschild BHs under different aspects. Kim and Kang [29] and Jimenez 
Madrid and Gonzalez-Diaz [30] studied accretion of dark energy on a static BH and a Kerr-Newman BH. Sharif and Abbas [28] discussed the accretion on stringy charged BHs due to phantom energy.

Recently, the framework of accretion on general static spherical symmetric BHs has been presented by Bahamonde and Jamil [22]. We have extended this general formalism for some RBHs. We analyze the effect of the mass of a RBH by choosing different values of the EoS parameter. This paper is organized as follows: In Sect. 2, we derive a general formalism for a spherically static accretion process. In Sect. 3, we discuss some RBHs and for each case, we explain the critical radius, critical points, speed of sound, radial velocities profile, energy density, and the rate of change of the RBH mass. In the end, we conclude our results.

\section{General formalism for accretion}

The generalized static spherical symmetry is characterized by the following line element:

$$
\begin{aligned}
\mathrm{d} s^{2}= & -X(r) \mathrm{d} t^{2}+\frac{1}{Y(r)} \mathrm{d} r^{2} \\
& +Z(r)\left(\mathrm{d} \theta^{2}+\sin \theta^{2} \mathrm{~d} \phi^{2}\right),
\end{aligned}
$$

where $X(r)>0, Y(r)>0$, and $Z(r)>0$ are functions of $r$ only. The energy-momentum tensor is considered in terms of a perfect fluid which is isotropic and inhomogeneous and defined as follows:

$T_{\mu \nu}=(\rho+p) u_{\mu} u_{\nu}+p g_{\mu \nu}$,

where $p$ is the pressure, $\rho$ is the energy density, and $u^{\mu}$ is the four-velocity, which is given by

$u^{\mu}=\frac{\mathrm{d} x^{\mu}}{\mathrm{d} \tau}=\left(u^{t}, u^{r}, 0,0\right)$,

where $\tau$ is the proper time. $u^{\theta}$ and $u^{\phi}$ both are equal to zero due to spherical symmetry restrictions. Here the pressure, the energy density, and the four-velocity components are only functions of $r$. The normalization condition of the four-velocity must satisfy $u^{\mu} u_{\mu}=-1$, and we get

$u^{t}:=\frac{\mathrm{d} t}{\mathrm{~d} \tau}=\sqrt{\frac{u^{2}+Y}{X Y}}$

where $u=\mathrm{d} r / \mathrm{d} \tau=u^{r}$ [22], $u^{t}$ can be negative or positive due to the square root which represents the backward or forward in time conditions. However, $u<0$ is required for the accretion process, otherwise for any outward flows $u>0$. Both inward and outward flows are very important in astrophysics. One can assume that the fluid is determined by dark energy or any kind of dark matter. For a spherically symmetric BH, the proper dark energy model could be obtained by generalizing Michel's theory. In dark energy accretion, Babichev et al. [25] have introduced the above generalization of the Schwarzschild black hole. Similarly, some authors $[22,31]$ have extended this procedure for a generalized static spherically symmetric $\mathrm{BH}$. In these works, the equation of continuity plays an important role, which turns out to be

$(\rho+p) u \frac{X(r)}{Y(r)} \sqrt{u^{2}+Y(r)} Z(r)=A_{0}$,

where $A_{0}$ is the constant of integration. Using $u_{\mu} T_{\mu}^{\mu \nu}=0$, we obtain the continuity (or relativistic energy flux) equation

$u^{\mu} \rho_{, \mu}+(\rho+p) u_{; \mu}^{\mu}=0$.

Furthermore, we assume $p=p(\rho)$, a certain EoS in this case. After some calculations, the above equation becomes

$\frac{\rho^{\prime}}{\rho+p}+\frac{u^{\prime}}{u}+\frac{X^{\prime}}{2 X}+\frac{Y^{\prime}}{2 Y}+\frac{Z^{\prime}}{Z}=0$,

where a prime represents the derivative with respect to $r$. By integrating the last equation, we obtain

$u Z(r) \sqrt{\frac{X(r)}{Y(r)}} e^{\int \frac{\mathrm{d} \rho}{\rho+\rho(\rho)}}=-A_{1}$,

where $A_{1}$ is the constant of integration. By equating Eqs. (5) and (8), we get

$(\rho+p) \sqrt{\frac{X(r)}{Y(r)}} \sqrt{u^{2}+Y} e^{-\int \frac{\mathrm{d} \rho}{\rho+p(\rho)}}=-\frac{A_{0}}{A_{1}}=A_{3}$,

where $A_{3}$ is another constant, depending upon $A_{0}$ and $A_{1}$. Moreover, the equation of mass flux yields

$\rho u \sqrt{\frac{X(r)}{Y(r)}} Z(r)=A_{2}$,

where $A_{2}$ is the constant of integration. By using Eqs. (5) and (10), we obtain the following important relation:

$\frac{(\rho+p)}{\rho} \sqrt{\frac{X(r)}{Y(r)}} \sqrt{u^{2}+Y}=\frac{A_{1}}{A_{2}} \equiv A_{4}$,

where $A_{4}$ is arbitrary constant which depends on $A_{1}$ and $A_{2}$. Taking differentials of Eqs. (10) and (11) and some manipulation lead to 


$$
\begin{aligned}
& \left(V^{2}-\frac{u^{2}}{u^{2}+Y}\right) \frac{\mathrm{d} u}{u}+\left(\left(V^{2}-1\right)\left(\frac{X^{\prime}}{X}-\frac{Y^{\prime}}{Y}\right)\right. \\
& \left.+\frac{Z^{\prime}}{Z} V^{2}-\frac{Y^{\prime}}{2\left(u^{2}+Y\right)}\right) \mathrm{d} r=0 .
\end{aligned}
$$

In addition, we have introduced the variable

$V^{2} \equiv \frac{\mathrm{d} \ln \rho+p}{\mathrm{~d} \ln \rho}-1$.

If the bracketed terms in Eq. (12) vanish, we obtain the critical point (where the speed of sound equals the speed of the flow), which is located at $r=r_{c}$. Hence at the critical point, we get

$V_{c}^{2}=\frac{u_{c}^{2}}{u_{c}^{2}+Y\left(r_{c}\right)}$

and Eq. (12) turns out to be

$$
\begin{aligned}
& \left(V_{c}^{2}-1\right)\left(\frac{X^{\prime}\left(r_{c}\right)}{X\left(r_{c}\right)}-\frac{Y^{\prime}\left(r_{c}\right)}{Y\left(r_{c}\right)}\right)+\frac{Z^{\prime}\left(r_{c}\right)}{Z\left(r_{c}\right)} V_{c}^{2} \\
& =\frac{Y^{\prime}\left(r_{c}\right)}{2\left(u_{c}^{2}+Y\left(r_{c}\right)\right)} .
\end{aligned}
$$

Also, $u_{c}$ is the critical speed of the flow evaluated at the critical value $r=r_{c}$. We can decouple the above two equations and obtain

$$
\begin{aligned}
u_{c}^{2}= & \frac{Y\left(r_{c}\right) Z\left(r_{c}\right) X^{\prime}\left(r_{c}\right)}{2 X\left(r_{c}\right) Z^{\prime}\left(r_{c}\right)}, \\
& V_{c}^{2}=\frac{Z\left(r_{c}\right) X^{\prime}\left(r_{c}\right)}{2 X\left(r_{c}\right) Z^{\prime}\left(r_{c}\right)+Z\left(r_{c}\right) X^{\prime}\left(r_{c}\right)} .
\end{aligned}
$$

The speed of sound is evaluated at $r=r_{c}$ as follows:

$c_{s}^{2}=\left.\frac{\mathrm{d} p}{\mathrm{~d} \rho}\right|_{r=r_{c}}=A_{4} \sqrt{\frac{Y\left(r_{c}\right)}{X\left(r_{c}\right)\left(u_{c}^{2}+Y\left(r_{c}\right)\right)}}-1$.

Obviously, $u_{c}^{2}$ and $V_{c}^{2}$ can never be negative and hence

$\frac{X^{\prime}\left(r_{c}\right)}{Z^{\prime}\left(r_{c}\right)}>0$

Moreover, the rate of change of the $\mathrm{BH}$ mass can be defined as follows [31]:

$\dot{M}_{\mathrm{acc}}=4 \pi A_{3} M^{2}(\rho+p)$.

Here a dot is for a derivative with respect to time. We can observe that the mass of the BH will increase for the fluid, $\rho+p>0$, and hence the accretion occurs outside the $\mathrm{BH}$. Otherwise, for $\rho+p<0$ like a fluid, the mass of the $\mathrm{BH}$ will decrease. The mass of the $\mathrm{BH}$ cannot remain fixed because it will decrease due to Hawking radiation, while it will increase due to accretion. If we consider the time dependence of the BH mass, then we first assume that it will not change the geometry and symmetry of space-time. Hence the space-time metric remains static spherically symmetric [22].

\section{Spherically symmetric metrics with charged RBHs}

In this section, we discuss the spherically symmetric metrics with charged RBHs in which $X(r)=Y(r)$. For this assumption, Eq. (16) gives

$$
\begin{gathered}
u_{c}^{2}=\frac{Z\left(r_{c}\right) X^{\prime}\left(r_{c}\right)}{2 Z^{\prime}\left(r_{c}\right)}, \\
V_{c}^{2}=\frac{Z\left(r_{c}\right) X^{\prime}\left(r_{c}\right)}{2 X\left(r_{c}\right) Z^{\prime}\left(r_{c}\right)+Z\left(r_{c}\right) X^{\prime}\left(r_{c}\right)} .
\end{gathered}
$$

Although our focus is on charged RBHs metrics with event horizons, the present analysis is forbidden for a horizon space-time. In many cases, we are concerned with critical values (critical radius), critical velocities, speed of sound in fluid, behavior of energy density of fluid, radial velocity, and the rate of change of the mass of the accreting objects. So the horizon is not involved anywhere [22].

\subsection{Charged RBH using Fermi-Dirac distribution}

The said RBH solution has the following metric functions [32]:

$X(r)=1-\frac{2 M}{r}\left(\frac{\xi(x)(\beta r)}{\xi_{\infty}}\right)^{\beta}=Y(r)$,

where the Fermi-Dirac distribution function is

$\xi(x)=\frac{1}{e^{x}+1}$.

By replacing $x=\frac{q^{2}}{M \beta r}$, we can obtain the distribution function as

$\xi(\beta r)=\frac{1}{e^{\frac{q^{2}}{M \beta r}}+1}$,

with normalization factor $\xi_{\infty}=\frac{1}{2}$. Also the distribution function satisfies

$\frac{\xi(r)}{\xi_{\infty}} \rightarrow 1$,

where $r \rightarrow \infty$. Hence the metric functions turn out to be

$$
X(r)=Y(r)=1-\frac{2 M}{r}\left(\frac{2}{e^{\frac{q^{2}}{M r}}+1}\right)^{\beta}, \quad Z(r)=r^{2} .
$$




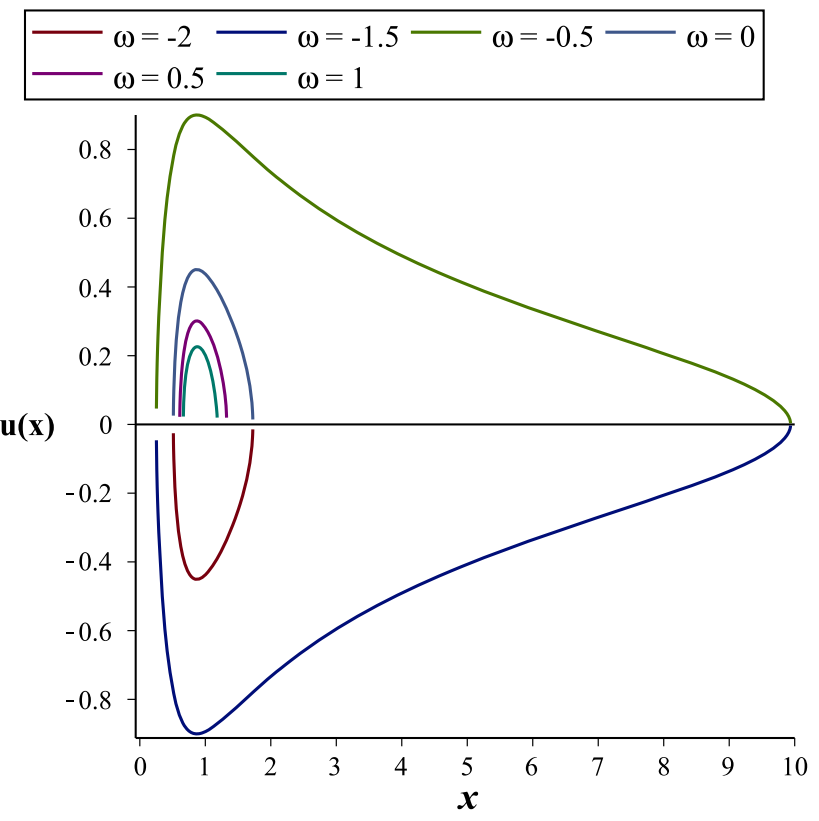

Fig. 1 Velocity profile against $x=\frac{r}{M}$ for $\beta=1, q=1.055 M$, $M=1$, and $A_{4}=0.45$ of the charged RBH using the Fermi-Dirac distribution

If we set $\beta \rightarrow 0$ and $\beta \rightarrow \infty$, we obtain

$$
\begin{aligned}
& X(r)=Y(r)=1-\frac{2 M}{r} e^{\frac{-q^{2}}{M r}}, \\
& X(r)=Y(r)=1-\frac{2 M}{r} e^{\frac{-q^{2}}{2 M r}} .
\end{aligned}
$$

In both equations, the difference of the factor 2 must be noted [32].

It is possible to integrate the conversation laws and obtain analytical expressions of the physical parameters. For simplicity, we will study the barotropic case where the fluid has the equation $p(r)=\omega \rho(r)$. Using (5) and (11), we obtain

$$
=\frac{\left(\left(2 M\left(\frac{1}{2} e^{\frac{q^{2}}{M r}}+\frac{1}{2}\right)^{-\beta}-r\right)(\omega+1)^{2}+A_{4}^{2} r\right)^{1 / 2}}{(\omega+1) \sqrt{r}},
$$

$$
\rho(r)=\frac{A_{2}(\omega+1)}{r^{3 / 2} \sqrt{\left(2 M\left(\frac{1}{2} e^{\frac{q^{2}}{M r}}+\frac{1}{2}\right)^{-\beta}-r\right)(\omega+1)^{2}+A_{4}^{2} r}} .
$$

The velocity profile for different values of $\omega$ is shown in Fig. 1 . Here $\omega=1,0,-1$ refer to the stiff, dust, and cosmological constant cases, respectively, and $-1<\omega<$

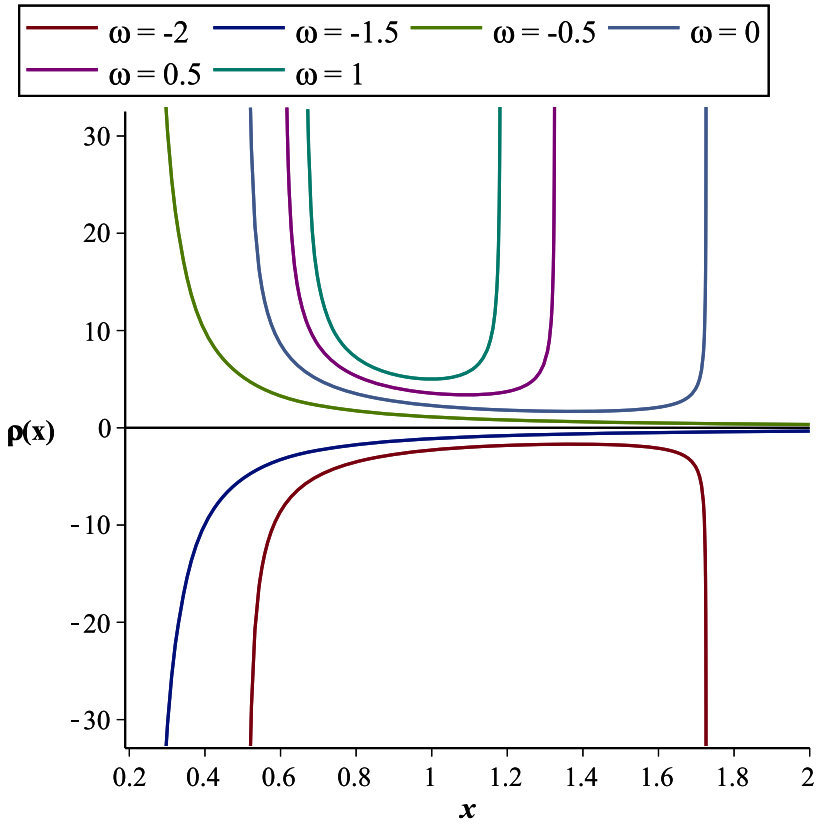

Fig. 2 Energy density against $x=\frac{r}{M}$ for $\beta=1, q=1.055 M, M=1$, $A_{2}=1$, and $A_{4}=0.45$ of the charged RBH using the Fermi-Dirac distribution

$-1 / 3$ and $\omega<-1$ refer to quintessence and phantom energy. It can be seen that for $\omega=-1.5,-2$ the radial velocity of the fluid is negative and it is positive for $\omega=-0.5,0,0.5,1$. If the flow is outward then $u<0$ is not allowed and vice versa. In the case of $\omega=-1.5,-0.5$ the fluid is at rest at $x=10$. Figure 2 represents the behavior of energy density of fluids in the surrounding area of the RBH. Obviously the WEC and DEC satisfied by dust, stiff, and quintessence fluids. When the phantom fluid $(\omega=-1.5,-2)$ moves toward the RBH then the energy density decreases and the reverse will happen for dust, stiff, and quintessence fluids $(\omega=-0.5,0,0.5,1)$. Asymptotically $\rho \rightarrow 0$ at infinity for $\omega=-1.5,-0.5$, while it approaches the maximum at $x=1.2,1.3,1.8$ and near the $\mathrm{RBH}$.

Using this metric, Eqs. (19) and (29), the rate of change of the mass of the RBH due to accretion becomes

$$
\dot{M}=\frac{4 \pi A_{2}^{2} A_{4}(\omega+1)}{r^{3 / 2} \sqrt{\left(2 M\left(\frac{1}{2} e^{\frac{q^{2}}{M r}}+\frac{1}{2}\right)^{-\beta}-r\right)(\omega+1)^{2}+A_{4}^{2} r}} .
$$

Figure 3 represents the change in RBH mass for different values of $\omega$. The mass of the RBH will increase near it and at $x=1.2,1.3,1.7$ for $\omega=1,0.5,0$, respectively. On the other hand, the mass of the RBH decreases near it and at $x=1.7$ for $\omega=-2$. Hence the mass of the RBH increases due to the accretion of quintessence, dust, and stiff matter, 


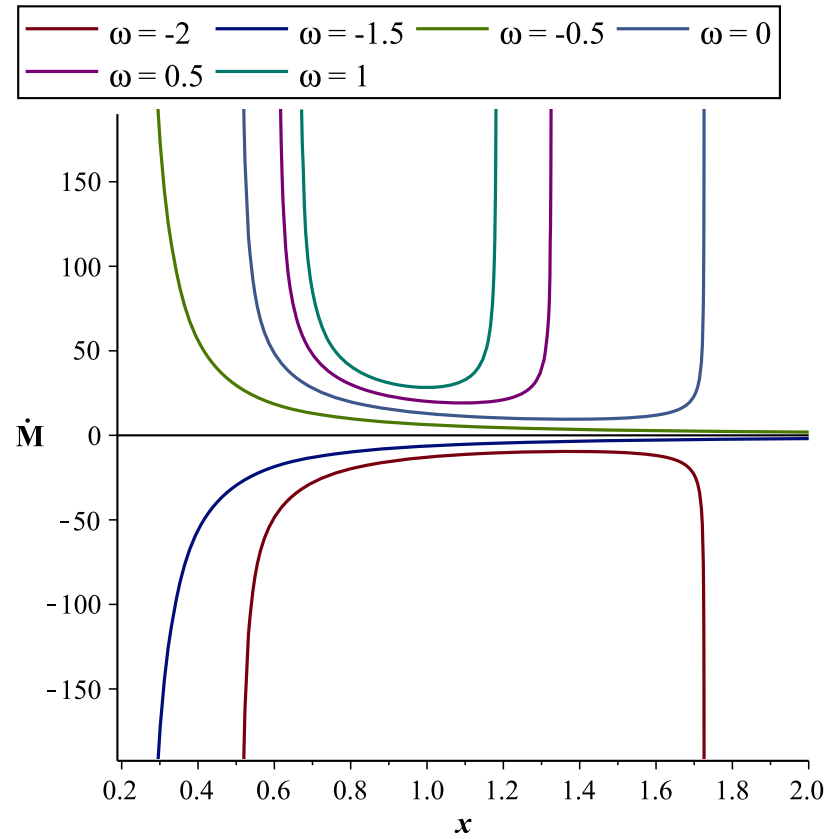

Fig. 3 Rate of change of the mass of a RBH against $x=\frac{r}{M}$ for $\beta=1$, $q=1.055 M, M=1, A_{2}=1$, and $A_{4}=0.45$ of a charged RBH using the Fermi-Dirac distribution

Table 1 Charged RBH using the Fermi-Dirac distribution

\begin{tabular}{llrc}
\hline$\omega$ & $r_{c}$ & $u\left(r_{c}\right)$ & \multicolumn{1}{l}{$c_{s}^{2}$} \\
\hline-2 & 1.37495 & 0.3138832070 & 0.0000002580 \\
-1.5 & 7.5044 & 0.2382908936 & -0.4999997476 \\
-0.5 & 7.5044 & -0.2382908936 & -0.4999997476 \\
0 & 1.3749 & -0.3138832070 & 0.0000002580 \\
0.5 & 1.092 & -0.2476468259 & 0.503110174 \\
1 & 0.999 & -0.1998921298 & 1.002986469 \\
\hline
\end{tabular}

while it decreases due to the accretion of phantom-like fluids.

The critical values, critical velocities, and speed of sound are obtained for different values of the EoS parameter in Table 1 . The critical radius is shifting to the left when $\omega \geq$ 0 increases. Thus, the in-falling fluid acquires supersonic speeds closer to $\mathrm{RBH}$. The same critical radius is obtained for $\omega=-2,0$ and $\omega=-1.5,-0.5$ with the same critical velocities but in an opposite direction. We get a negative speed of sound at $x=7.5044$ and a positive speed of sound for the remaining critical radius. Also, the speed of sound increases near the RBH. For this metric, we find that

$$
u_{c}^{2}=\frac{2^{\beta-1}\left(\left(M r-q^{2}\right) e^{\frac{q^{2}}{\beta M r}}+M r\right)}{r^{2}\left(e^{\frac{q^{2}}{\beta M r}}+1\right)^{\beta+1}}
$$

$$
V_{c}^{2}=\frac{2^{\beta}\left(\left(M r-q^{2}\right) e^{\frac{q^{2}}{\beta M r}}+M r\right)}{2 r^{2}\left(e^{\frac{q^{2}}{\beta M r}}+1\right)^{\beta+1}-2^{\beta}\left(\left(3 M r+q^{2}\right) e^{\frac{q^{2}}{\beta M r}}+3 M r\right)} .
$$

Also, the condition (18) yields

$$
\frac{2^{\beta}\left(\left(M r-q^{2}\right) e^{\frac{q^{2}}{\beta M r}}+M r\right)}{r^{4}\left(e^{\frac{q^{2}}{\beta M r}}+1\right)^{\beta+1}}>0
$$

\subsection{Charged RBH using logistic distribution}

The logistic distribution function is [32]

$\xi(x)=\frac{e^{-x}}{\left(e^{-x}+1\right)^{2}}$,

in which we replace $x=\frac{2 q^{2}}{M \beta r}$; then we obtain the distribution function

$\xi(\beta r)=\frac{e^{\frac{-2 q^{2}}{M \beta r}}}{\left(e^{\frac{-2 q^{2}}{M \beta r}}+1\right)^{2}}$,

with normalization factor $\sigma_{\infty}=\frac{1}{4}$. Also the distribution function satisfies

$\frac{\xi(r)}{\xi_{\infty}} \rightarrow 1$

where $r \rightarrow \infty$. The horizons can be obtained for $\beta=1$ where $q=1.055 M$. The metric function can be written as

$X(r)=Y(r)=1-\frac{2 M}{r}\left(\frac{4 e^{-\sqrt{\frac{2 q^{2}}{\beta M r}}}}{\left(e^{-\sqrt{\frac{2 q^{2}}{\beta M r}}}+1\right)^{2}}\right)^{\beta}$,

$Z(r)=r^{2}$.

If we set $\beta \rightarrow 0$, then we obtain the Schwarzschild $\mathrm{BH}$, and if we set $\beta \rightarrow \infty$ we get

$X(r)=Y(r)=1-\frac{2 M}{r} e^{\frac{-q^{2}}{2 M r}}$.

It is noteworthy that this metric function corresponds to an Ayon-Beato and Garca BH [32].

The radial velocity and energy density for the metric (37) using Eqs. (5) and (10) is given by 


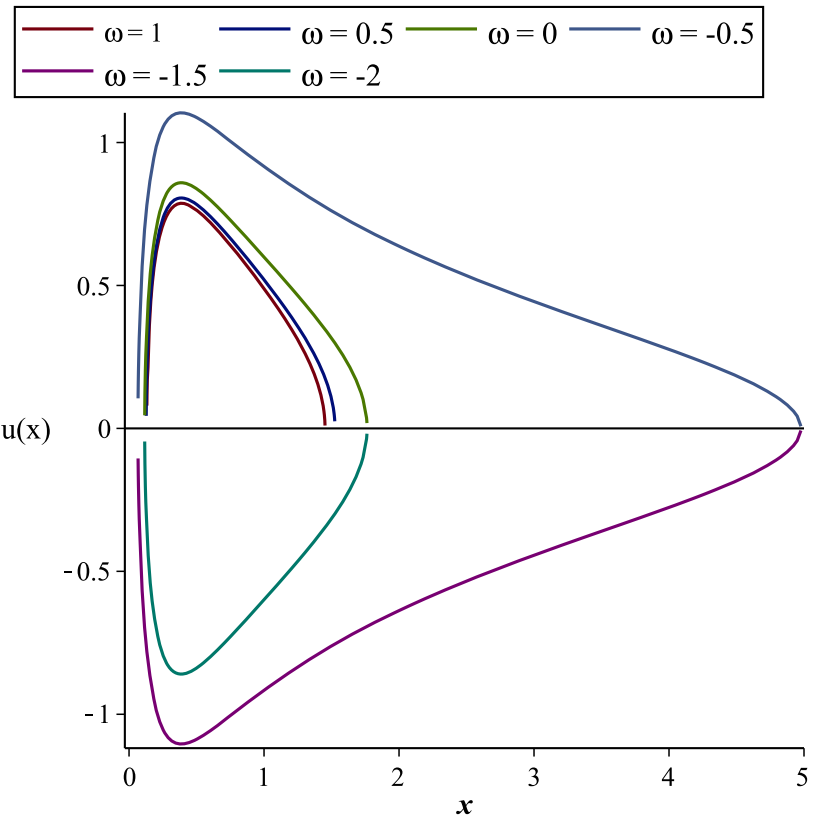

Fig. 4 Velocity profile against $x=\frac{r}{M}$ for $\beta=1, q=1.055 M$, $M=1$, and $A_{4}=0.4$ of a charged $\mathrm{RBH}$ using the logistic distribution

$$
\begin{aligned}
u(r)= & \frac{1}{(\omega+1) \sqrt{r}} \\
& \times\left(\left(-2 M\left(\frac{4 e^{-\sqrt{\frac{2 q^{2}}{\beta M r}}}}{\left(e^{-\sqrt{\frac{2 q^{2}}{\beta M r}}}+1\right)^{2}}\right)^{\beta}+r\right)(\omega+1)^{2}-A_{4}^{2} r\right)^{1 / 2},
\end{aligned}
$$

$$
\rho(r)=-\frac{A_{2}(\omega+1)}{r^{3 / 2}\left(\left(2 M\left(\frac{4 e^{-\sqrt{\frac{2 q^{2}}{\beta M r}}}}{\left(e^{-\sqrt{\frac{2 q^{2}}{\beta M r}}}+1\right)^{2}}\right)^{\beta}-r\right)(\omega+1)^{2}+A_{4}^{2} r\right)^{1 / 2} .}
$$

The velocity profile for different values of $\omega$ is shown in Fig. 4. It can be observed that for $\omega=-1.5,-2$ the radial velocity of the fluid is negative and it is positive for $\omega=-0.5,0,1$. If the flow is inward then $u>0$ is not allowed and vice versa. In the case of $\omega=-2,0$ the fluid is at rest at $x \approx 5$. Figure 5 represents the behavior of energy density of fluids in the surrounding area of the RBH. Obviously the WEC and DEC are satisfied by dust, stiff, and quintessence fluids. When a phantom-like fluid $(\omega=-1.5,-2)$ moves toward a RBH the energy density decreases and the reverse will happen for dust, stiff, and quintessence fluids $(\omega=-0.5,0,0.5,1)$.

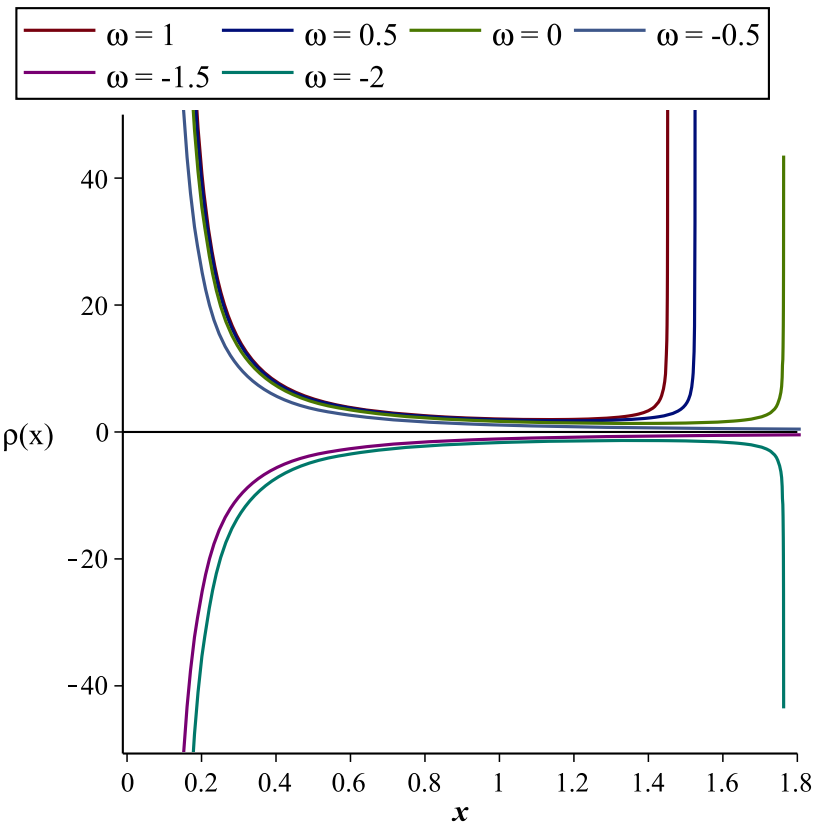

Fig. 5 The energy density against $x=\frac{r}{M}$ for $\beta=1, q=1.055 M$, $M=1, A_{2}=1$, and $A_{4}=0.4$ of a charged RBH using logistic distribution

The $\dot{M}$ of an RBH for distinct EoS parameters is obtained by using (19),

$$
\left.\left.\left.=-\frac{4 \pi A_{2}^{2} A_{4}(\omega+1)}{r^{3 / 2}\left(\left(2 M\left(\frac{4 e^{-\sqrt{\frac{2 q^{2}}{\beta M r}}}}{\left(e^{-\sqrt{\frac{2 q^{2}}{\beta M r}}}+1\right.}\right)^{2}\right.\right.}\right)^{\beta}-r\right)(\omega+1)^{2}+A_{4}^{2} r\right)^{1 / 2} .
$$

Figure 6 represents the change in the $\mathrm{RBH}$ mass against $x$. It is evident that the mass of the RBH increases due to quintessence, dust, and stiff fluids and it decreases due to phantom fluids.

The critical radius, the critical velocity, and the speed of sound are obtained for different values of EoS parameter in Table 2. The critical radius is shifting to the right when $\omega \geq 0$ increases. Thus the in-falling fluid acquires supersonic speeds closer to the RBH. For a phantom-like fluid, quintessence, dust, and stiff matter the critical radius and critical velocities are explained in Table 2. Same critical radius is obtained for $\omega=-2,0$ and $\omega=-1.5,-0.5$ with the same critical velocities but different in sign. We obtained a negative speed of sound at $x=1.36375,3.777412$ and positive speed of sound at $x=1.12974,1.1850$. Near the RBH the speed of sound will increase. For this metric we find that 


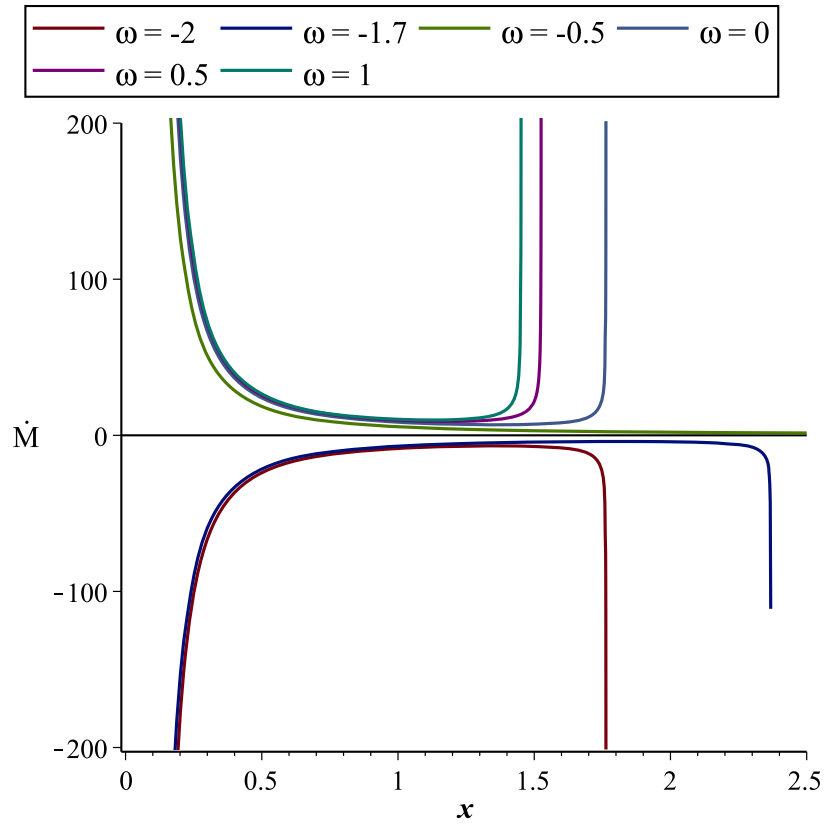

Fig. 6 Rate of change of the mass of a RBH against $x=\frac{r}{M}$ for $\beta=1$, $q=1.055 M, M=1, A_{2}=1$, and $A_{4}=0.4$ of a charged RBH using the logistic distribution

Table 2 Charged RBH using logistics distribution

\begin{tabular}{llll}
\hline$\omega$ & $r_{c}$ & $u\left(r_{c}\right)$ & \multicolumn{1}{l}{$c_{s}^{2}$} \\
\hline-2 & 1.36375 & -0.3998729763 & -0.1018620364 \\
-1.5 & 3.777412 & -0.3138895411 & -0.5007414180 \\
-0.5 & 3.77412 & 0.3138895411 & -0.5007414180 \\
0 & 1.36375 & 0.3998724197 & -0.1018620364 \\
0.5 & 1.1850 & 0.4018068205 & 0.116622918 \\
1 & 1.12974 & 0.4014558621 & 0.231766770 \\
\hline
\end{tabular}

Here the function

$M(r)=M\left(1-\tan h\left(\frac{q^{2}}{2 M r}\right)\right)$,

and its associated electric field source is

$$
\begin{aligned}
E= & \frac{q}{r^{2}}\left(1-\tan h^{2}\left(\frac{q^{2}}{2 M r}\right)\right) \\
& \times\left(1-\frac{q^{2}}{4 M r} \tan h\left(\frac{q^{2}}{2 M r}\right)\right),
\end{aligned}
$$

Also, the condition (18) yields

$$
\frac{2^{-1+2 \beta} \sqrt{M} e^{-\sqrt{\frac{2 q^{2} \beta}{M r}}}\left((\sqrt{2} q \beta+2 \sqrt{\beta M r}) e^{-\sqrt{\frac{2 q^{2} \beta}{M r}}}+(-\sqrt{2} q \beta+2 \sqrt{\beta M r})\right)}{\sqrt{\beta} r^{7 / 2}\left(e^{\sqrt{\frac{2 q^{2} \beta}{M r}}}\right)}>0 .
$$

\subsection{Charged RBH from nonlinear electrodynamics}

We use the line element

$$
X(r)=Y(r)=1-\frac{2 M(r)}{r} .
$$

where $q$ and $M$ represent the electric charge and the mass, respectively [33]. The solution elaborates $\mathrm{RBH}$ and its global structure is like R-N BH. The asymptotic behavior of the solution is

$$
X(r)=1-\frac{2 M}{r}+\frac{q^{2}}{r^{2}}+O\left(\frac{1}{r^{4}}\right)
$$




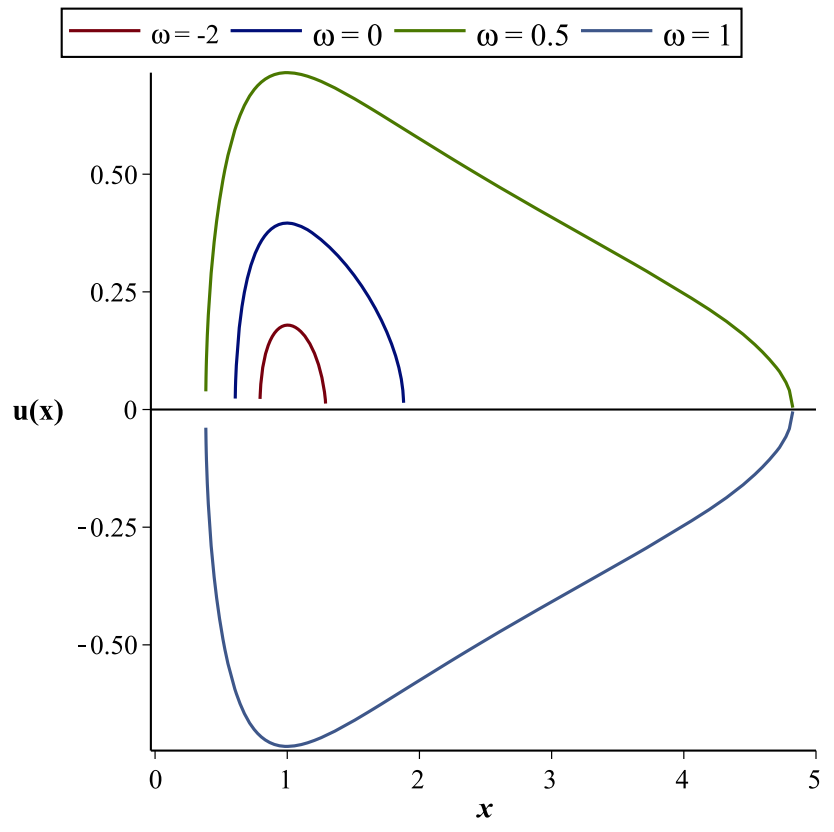

Fig. 7 Velocity profile against $x=\frac{r}{M}$ for $q=1.055 M, M=1$, and $A_{4}=0.7$ of a charged RBH from nonlinear electrodynamics

So we have the metric function

$$
\begin{aligned}
X(r) & =Y(r) \\
& =1-\frac{2 M}{r}\left(M\left(1-\tan h\left(\frac{q^{2}}{2 M r}\right)\right)\right), \quad Z(r)=r^{2} .
\end{aligned}
$$

The radial velocity and energy density for this metric are given by

$u(r)=\frac{\sqrt{\left(-2 M \tan h\left(\frac{q^{2}}{2 M r}\right)+2 M-r\right)(\omega+1)^{2}+A_{4}^{2} r}}{(\omega+1) \sqrt{r}}$,

$$
\rho(r)=\frac{(\omega+1) A_{2}}{r^{3 / 2} \sqrt{\left(-2 M \tan h\left(\frac{q^{2}}{2 M r}+2 M-r\right)\right)(\omega+1)^{2}+A_{4}^{2} r}} .
$$

The absolute value of the velocity profile for different values of $\omega$ is shown in Fig. 7. It can be observed that for $\omega=-2$ the radial velocity of the fluid is negative and it is positive for $\omega=0.5,0,1$. If the flow is inward then $u>0$ is not allowed and vice versa. In the case of $\omega=-2,0$ the fluid is at rest at $x \approx 5$. Figure 8 represents the energy density of fluids in the region of the RBH. It is apparent that the WEC and DEC is satisfied by phantom fluids. When the phantom fluids moves toward the RBH the energy density increases; on the other hand it decreases for dust and stiff matter.

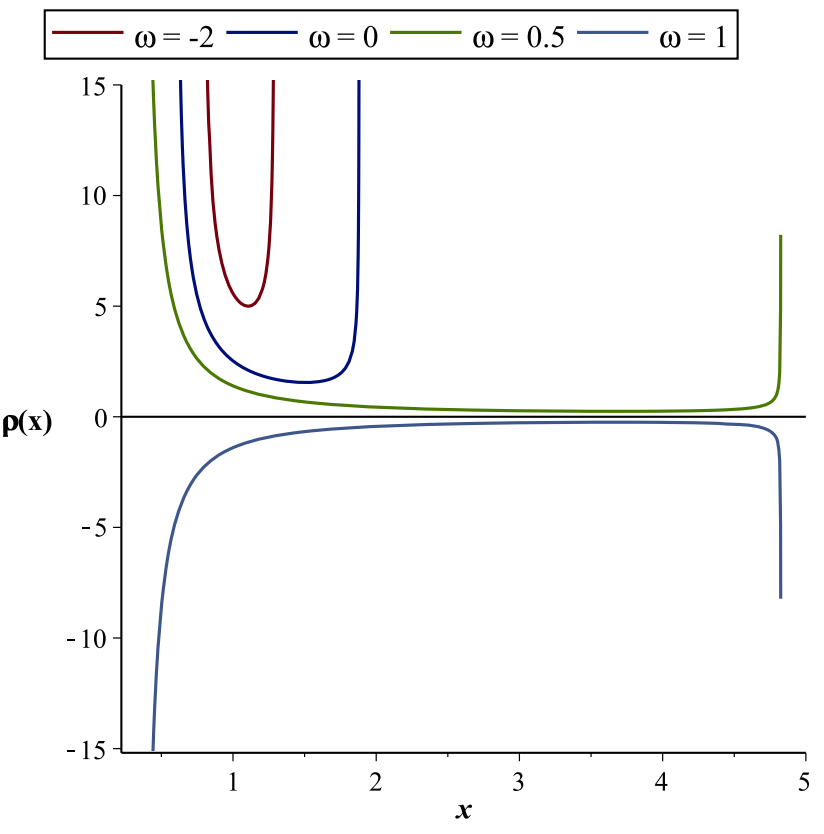

Fig. 8 Energy density against $x=\frac{r}{M}$ for $q=1.055 M, M=1, A_{2}=$ 1 , and $A_{4}=0.7$ of a charged RBH from nonlinear electrodynamics

The rate of change of the mass is given by

$$
\dot{M}=-\frac{4 \pi A_{2}^{2} A_{4}(\omega+1)}{r^{3 / 2} \sqrt{\left(-2 M \tan h\left(\frac{q^{2}}{2 M r}+2 M-r\right)\right)(\omega+1)^{2}+A_{4}^{2} r}} .
$$

The rate of change of in the RBH mass against $x$ is plotted in Fig. 9. Due to accretion of dust and stiff matter the mass of the RBH will increase for small values of $x$ and vice versa for phantom fluids. It is also noted that the maximum rate of the RBH mass increases due to $\omega=1$ followed by $\omega=$ $0.5,0,-2$.

The critical values, critical velocities, and speed of sound are obtained for different values of the EoS parameter in Table 3. The critical radius is shifting to the right when $\omega \geq 0$ increases. The speed of sound is negative at $x=3.685523529$ and near the $\mathrm{BH}$ the speed of sound will increase. For this RBH we find that

$u_{c}^{2}=\frac{q^{2}\left(\tan h^{2}\left(\frac{q^{2}}{2 M r}\right)-1\right)+2 M r\left(-\tan h\left(\frac{q^{2}}{2 M r}\right)+1\right)}{4 r^{2}}$,

$$
V_{c}^{2}=\frac{q^{2}\left(\tan h^{2}\left(\frac{q^{2}}{2 M r}\right)-1\right)+2 M r\left(-\tan h\left(\frac{q^{2}}{2 M r}\right)+1\right)}{4 r^{2}+q^{2}\left(\tan h^{2}\left(\frac{q^{2}}{2 M r}\right)-1\right)+6+M r\left(\tan h\left(\frac{q^{2}}{2 M r}\right)-1\right)} .
$$




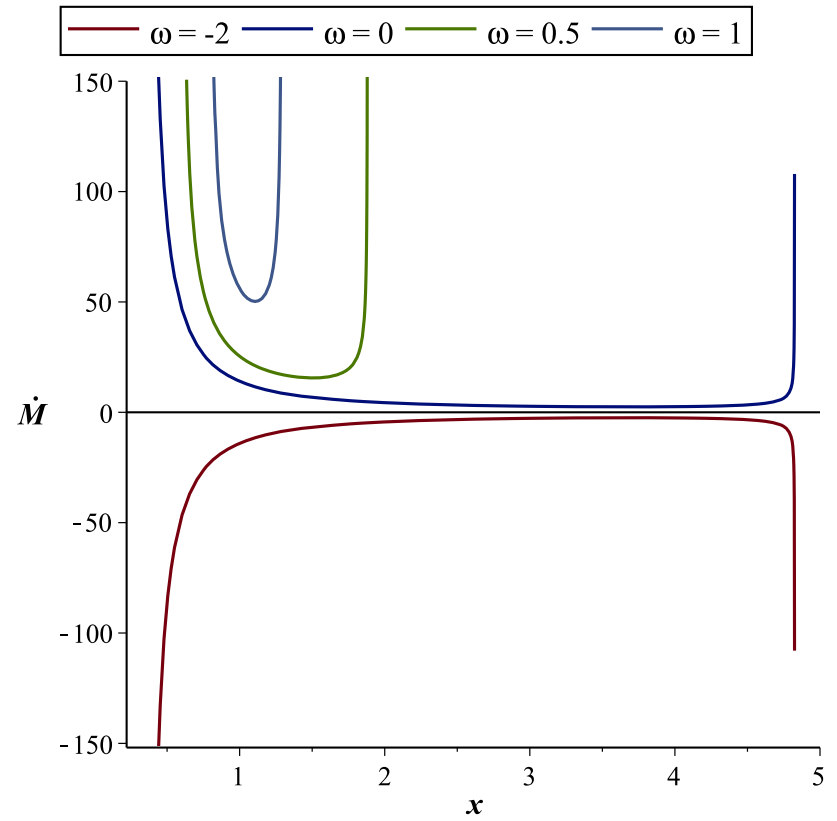

Fig. 9 Rate of change of the mass of an RBH against $x=\frac{r}{M}$ for $q=1.055 M, M=1, A_{2}=1$, and $A_{4}=0.7$ of charged $\mathrm{RBH}$ from nonlinear electrodynamics

Table 3 Charged RBH from nonlinear electrodynamics

\begin{tabular}{llrl}
\hline$\omega$ & $r_{c}$ & $u\left(r_{c}\right)$ & \multicolumn{1}{l}{$c_{s}^{2}$} \\
\hline-2 & 3.685523529 & -0.2993097288 & -0.125 \\
0 & 3.685523529 & 0.2993097288 & -0.125 \\
0.5 & 1.506050868 & 0.2844719573 & 0.312500584 \\
1 & 1.106971797 & 0.1633564212 & 0.750003072 \\
\hline
\end{tabular}

Also, the condition (18) yields

$$
\frac{\left(\tan h^{2}\left(\frac{q^{2}}{2 M r}\right)-1\right) q^{2}+2 M r\left(-\tan h\left(\frac{q^{2}}{2 M r}\right)+1\right)}{2 r^{4}}>0 \text {. }
$$

\subsection{Kehagias-Sftesos asymptotically flat BH}

KS studied the following BH metric:

$X(r)=Y(r)=1+b r^{2}-\sqrt{b^{2} r^{4}+4 M b r}, \quad Z(r)=r^{2}$.

In the frame work of Horava theory, where $m$ is the mass, $b$ is the positive constant related to the coupling constant of the theory. The metric asymptotically behaves like the usual Schwarzschild BH [34],

$$
X(r)=Y(r) \approx 1-\frac{2 M}{r}+O\left(\frac{1}{r^{4}}\right),
$$

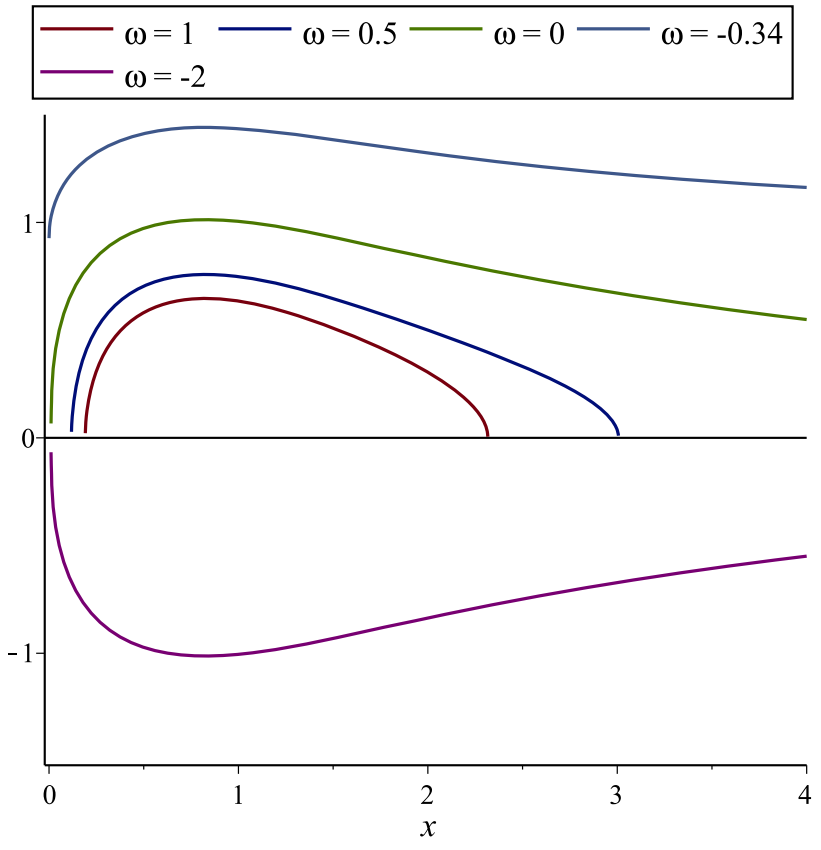

Fig. 10 Velocity profile against $x=\frac{r}{M}$ for $M=1, b=0.9$ and $A_{4}=0.9$ of a Kehagias-Sftesos asymptotically flat $\mathrm{BH}$

for $r \gg\left(\frac{r}{b}\right)^{1 / 3}$. The KS metric has two horizons at

$r_{ \pm}=M\left(1 \pm \sqrt{\left(1-\frac{1}{2 b M^{2}}\right)}\right)$,

with $2 b M^{2} \geq 1$ [34].

The radial velocity and energy density are given by

$u(r)=\frac{\left(A_{4}^{2}+\left(\sqrt{b^{2} r^{4}+4 M b r}-b r^{2}-1\right)(\omega+1)^{2}\right)^{1 / 2}}{\omega+1}$,

$\rho(r)=\frac{A_{2}(\omega+1)}{r^{2}\left(A_{4}^{2}+\left(\sqrt{b^{2} r^{4}+4 M b r}-b r^{2}-1\right)(\omega+1)^{2}\right)^{1 / 2}}$.

The radial velocity for different values of $\omega$ is shown in Fig. 10. The radial velocity is negative for a phantom-like fluid and positive for quintessence, dust, and stiff matter. The evolution of the energy density of the fluids in the surrounding area of an RBH is plotted in Fig. 11. The energy density for phantom fluids is negative, while the energy density for stiff, dust, and quintessence fluids is positive.

For this RBH, rate of change of the mass becomes

$$
\dot{M}=\frac{4 \pi A_{2}^{2} A_{4}(\omega+1)}{r^{2}\left(A_{4}^{2}+\left(\sqrt{b^{2} r^{4}+4 M b r}-b r^{2}-1\right)(\omega+1)^{2}\right)^{1 / 2}} .
$$




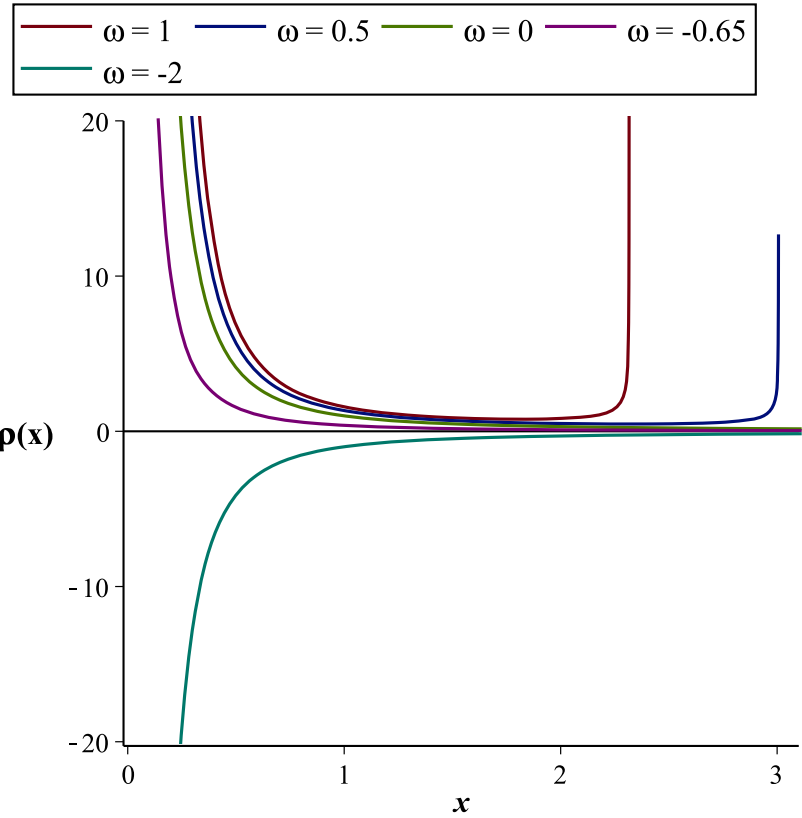

Fig. 11 Energy density against $x=\frac{r}{M}$ for $M=1, A_{2}=1, b=0.9$, and $A_{4}=0.9$ of the Kehagias-Sftesos asymptotically flat BH

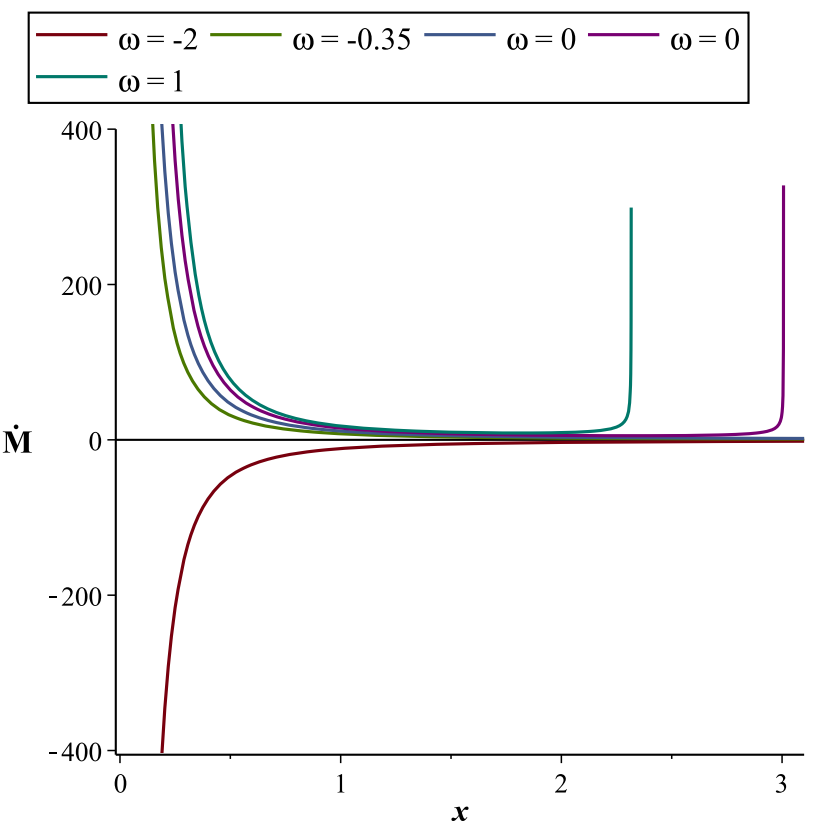

Fig. 12 Rate of change of the mass of an RBH against $x=\frac{r}{M}$ for $M=1, A_{2}=1, b=0.9$, and $A_{4}=0.9$ of a Kehagias-Sftesos asymptotically flat $\mathrm{BH}$

Figure 12 represents the rate of change in an RBH mass against $x$. We see that the RBH mass will increase for $\omega=$ $-0.35,0,0.5,1$, and it will decrease for $\omega=-2$.

The critical values, critical velocities, and speeds of sound for different values of $\omega$ are presented in Table 4. For quintessence matter, we obtain a very large critical radius. Similarly to the case before, we obtain the same critical radius
Table 4 Kehagias-Sftesos asymptotically flat BH

\begin{tabular}{llcr}
\hline$\omega$ & $r_{c}$ & $u\left(r_{c}\right)$ & \multicolumn{1}{c}{$c_{s}^{2}$} \\
\hline-2 & 7.8946 & -0.2505321935 & 0.0000008330 \\
-0.34 & 30267.74 & 0.6327458490 & -0.0513167023 \\
0 & 7.8946 & 0.2505321736 & 0.0000008330 \\
0.5 & 2.3185 & 0.3961993774 & 0.500013404 \\
1 & 1.8183 & 0.3888079314 & 0.500013404 \\
\hline
\end{tabular}

for dust and phantom-like fluids and the same critical velocities but different in sign. If we increase the EoS parameter then the critical radius is shifted near RBH. It is evident that the critical velocity is negative for a phantom-like fluid and positive for quintessence, dust, and stiff matter. The speed of sound is negative at $x=30267.74$ and positive for the remaining critical radius. For this metric, we find that

$$
\begin{aligned}
& u_{c}^{2}=\frac{r}{4}\left(2 b r-\frac{2 b^{2} r^{3}+2 M b}{\sqrt{r\left(b^{2} r^{3}+4 M b\right)}}\right), \\
& V_{c}^{2}=\frac{r^{2}\left(2 b r-\frac{2 b^{2} r^{3}+2 M b}{\sqrt{r\left(b^{2} r^{3}+4 M b\right)}}\right)}{r^{2}\left(2 b r-\frac{2 b^{2} r^{3}+2 M b}{\sqrt{r\left(b^{2} r^{3}+4 M b\right)}}\right)+4 r\left(1+b r^{2}-\sqrt{r\left(b^{2} r^{3}+4 M b\right)}\right)} .
\end{aligned}
$$

The condition (18) becomes

$$
\frac{\left(2 b r-\frac{2 b^{2} r^{3}+2 M b}{\sqrt{r\left(b^{2} r^{3}+4 M b\right)}}\right)}{2 r}>0 .
$$

\section{Concluding remarks}

In this work, we have investigated the accretion onto various RBHs (such as an RBH using the Fermi-Dirac distribution, a RBH using the logistic distribution, an RBH using nonlinear electrodynamics, and a Kehagias-Sftesos asymptotically flat RBH) which asymptotically leads to Schwarzschild and Reissner-Nordstrom BHs (most of them satisfy the WEC). We have followed the procedure of Bahamonde and Jamil [22] and obtained the critical points, critical velocities, and the behavior of the speed of sound for the chosen RBHs. Moreover, we have analyzed the behavior of the radial velocity, the energy density, and the rate of change of the mass for RBHs for various EoS parameters. For calculating these quantities, we have assumed the barotropic EoS and found the relationship between the conservation law and the barotropic EoS. We have found that the radial velocity $(u)$ of the fluid is positive for stiff, dust, and quintessence matter and it is 
negative for phantom-like fluids. If the flow is inward then $u<0$ is not allowed and $u>0$ is not allowed for outward flow. Also, we have seen that the energy density remains positive for quintessence, dust, and stiff matter, while it becomes negative for a phantom-like fluid near RBHs.

In addition, the rate of change of the mass of the $\mathrm{BH}$ is a dynamical quantity, so the analysis of the nature of its mass in the presence of various dark energy models may become very interesting in the present scenario. Also, the sensitivity (increasing or decreasing) of the BHs' mass depends upon the nature of the fluids which accrete onto it. Therefore, we have considered the various possibilities of accreting fluids, such as dust and stiff matter, quintessence, and phantom. We have found that the rate of change of the mass of all RBHs increases for dust and stiff matter, and quintessence-like fluids, since these fluids do not have enough repulsive force. However, the mass of all RBHs decreases in the presence of a phantom-like fluid (and the corresponding energy density and radial velocity become negative) because it has a strong negative pressure. This result shows the consistency with several works [22,31,35-47]. Also, this result favors the phenomenon that the universe undergoes the big rip singularity, where all the gravitationally bounded objects are dispersed due to the phantom dark energy.

Although we have assumed the presence of a static fluid, this may be extended for a non-static fluid without assuming any EoS and thus can be obtained more interesting results. This is left for future considerations.

Open Access This article is distributed under the terms of the Creative Commons Attribution 4.0 International License (http://creativecomm ons.org/licenses/by/4.0/), which permits unrestricted use, distribution, and reproduction in any medium, provided you give appropriate credit to the original author(s) and the source, provide a link to the Creative Commons license, and indicate if changes were made. Funded by SCOAP ${ }^{3}$.

\section{References}

1. S. Perlmutter et al. [Supernova Cosmology Project Collaboration], Astrophys. J. 517, 565 (1999)

2. D.N. Spergel et al. [WMAP Collaboration], Astrophys. J. Suppl. 170, 377 (2007)

3. D.J. Eisenstein et al. [SDSS Collaboration], Astrophys. J. 633, 560 (2005)
4. A.G. Riess, et al. [Supernova Search Team Collaboration], Astron. J. 116, 1009 (1998)

5. V.B. Johri, Phys. Rev. D 70, 041303 (2004)

6. F.S.N. Lobo, Phys. Rev. D 71, 084011 (2005)

7. S. Nojiri, S. Odintsov, Phys. Rep. 505, 59144 (2011)

8. C. Armendariz-Picon, V.F. Mukhanov, P.J. Steinhardt, Phys. Rev. Lett. 85, 4438 (2000)

9. M. Gasperini et al., Phys. Rev. D 65, 023508 (2002)

10. B. Gumjudpai, J. Ward, Phys. Rev. D 80, 023528 (2009)

11. J. Martin, M. Yamaguchi, Phys. Rev. D 77, 123508 (2008)

12. H. Wei, R.G. Cai, D.F. Zeng, Class. Quantum Gravity 22, 3189 (2005)

13. A. Sen, JHEP 0207, 065 (2002)

14. R.R. Caldwell, Phys. Lett. B 545, 23 (2002)

15. A.Y. Kamenshchik, U. Moschella, V. Pasquier, Phys. Lett. B 511, $265(2001)$

16. E.J. Copeland, M. Sami, S. Tsujikawa, Int. J. Mod. Phys. D 15, 1753 (2006)

17. E. Elizalde, S.R. Hildebrandt, Phys. Rev. D 65, 124024 (2002)

18. O.B. Zaslavskii, Phys. Lett. B 688, 278 (2010)

19. S.W. Hawking, G.F. Ellis, The Large Scale Structure of SpaceTime (Cambridge University Press, Cambridge, 1973)

20. J.M.M. Senovilla, Gen. Relativ. Gravit. 30, 701 (1998)

21. J. Bardeen, in Presented at GR5, Tiflis, U.S.S.R., and Published in the Conference Proceedings in the U.S.S.R. (1968)

22. S. Bahamonde, M. Jamil, Eur. Phys. J. C 75, 508 (2015)

23. H. Bondi, Mon. Not. R. Astron. Soc. 112, 195 (1952)

24. F.C. Michel, Astrophys. Space Sci. 15, 153 (1972)

25. E. Babichev et al., Phys. Rev. Lett. 93, 021102 (2004)

26. E. Babichev, V. Dokuchaev, Y. Eroshenko, J. Exp. Theor. Phys. 100, 528538 (2005)

27. M. Jamil, Eur. Phys. J. C 62, 609 (2009)

28. M. Sharif, G. Abbas, Chin. Phys. Lett. 29, 010401 (2012)

29. S.W. Kim, Y. Kang, Int. J. Mod. Phys. Conf. Ser. 12, 320 (2012)

30. J.A Jimenez Madrid, P.F. Gonzalez-Diaz, Gravit. Cosmol. 14, 213 (2008)

31. U. Debnath, Eur. Phys. J. C 75, 129 (2015)

32. L. Balart, E.C. Vagenas, Phys. Rev. D 90, 124045 (2014)

33. M. Sharif, A. Jawad, Mod. Phys. Lett. A 25, 3241-3250 (2010)

34. H. Culetu, Astrophys. Space Sci. 2, 360 (2015)

35. U. Debnath, Eur. Phys. J. C 75, 449 (2015)

36. H. Wei, Class. Quantum Gravity 29, 175008 (2012)

37. F.S.N. Lobo, Phys. Rev. D 71, 124022 (2005)

38. F.S.N. Lobo, Phys. Rev. D 71, 084011 (2005)

39. S. Sushkov, Phys. Rev. D 71, 043520 (2005)

40. E. Babichev, V. Dokuchaev, Y. Eroshenko, Phys. Rev. Lett. 93, 021102 (2004)

41. M. Sharif, G. Abbas, Chin. Phys. Lett. 28, 090402 (2011)

42. P. Martin-Moruno, Phys. Lett. B 659, 40 (2008)

43. M. Jamil, M.A. Rashid, A. Qadir, Eur. Phys. J. C 58, 325 (2008)

44. E. Babichev et al., Phys. Rev. D 78, 104027 (2008)

45. M. Jamil, Eur. Phys. J. C 62, 325 (2009)

46. M. Jamil, A. Qadir, Gen. Relativ. Gravit. 43, 1069 (2011)

47. J. Bhadra, U. Debnath, Eur. Phys. J. C 72, 1912 (2012) 